\title{
Falla renal aguda por rabdomiólisis; reporte de caso en un paciente pediátrico con múltiples picaduras de abejas
}

\author{
Acute kidney failure due to rhabdomyolysis; case report in a pediatric patient with multiple \\ bee stings
}

\author{
León F. Mendoza-Vega ${ }^{1 *}$, Yesica Guillestegui-Rodríguez ${ }^{2}$ y Elena I. Pasos-Solís ${ }^{2}$ \\ ${ }^{1}$ Departamento de División de Medicina Crítica; ${ }^{2}$ Departamento de Pediatría. Hospital Infantil de Tlaxcala, Apetatitlán, Tlaxcala, México
}

\section{Introducción}

Las picaduras de abejas o insectos del orden de los himenópteros tienen una presentación clínica muy variable, debido a que el efecto del veneno puede ser local o sistémico'. La mayoría de las muertes están relacionadas con reacciones de hipersensibilidad inmediata, causando anafilaxia ${ }^{2}$. En todo el mundo se reportan hasta 100,000 muertes anuales, con mayor incidencia en las regiones tropicales ${ }^{3}$, y el riesgo de muerte aumenta hasta en un $25 \%$ con la presencia de falla renal aguda 4 .

La presentación con afectación renal, como la rabdomiólisis, está catalogada como una de las manifestaciones inusuales en los niños; sin embargo, en los adultos se reporta hasta en un $30 \%$ de los casos y es de gran relevancia su detección oportuna para un tratamiento adecuado 5 .

El principal componente del veneno de las abejas es la melitina, que desorganiza las membranas celulares, libera aminas biogénicas, altera la permeabilidad celular y genera liberación de histamina (causante del dolor local y de las reacciones anafilácticas) ${ }^{6}$. También induce liberación de catecolaminas, que en conjunto con la fosfolipasa A2 causa hemólisis intravascular, lo que puede ocasionar rabdomiólisis y falla renal aguda por vasoconstricción renal, formación de depósitos de mioglobina intratubular y citotoxicidad directa de la mioglobina a los túbulos renales; complicaciones encontradas en casos con múltiples picaduras, por lo general más de $500^{1,7}$.

Los valores de creatina cinasa son el indicador más sensible de daño al miocito en la rabdomiólisis, incrementándose 12 horas después del inicio del daño muscular, con picos a los 1-3 días y descenso en 3-5 días después de la remisión de la alteración muscular?.

Reportamos un caso atendido en un hospital infantil, de un paciente que desarrolló intoxicación por picadura de abejas, rabdomiólisis y falla renal aguda, mostrando sus características clínicas, tratamiento y evolución clínica hasta su egreso.

\section{Descripción del caso}

Varón de 13 años, sin antecedentes de importancia. Es referido desde una clínica privada posterior a sufrir picaduras de abejas, 22 horas previas al ingreso. Ignoramos la cantidad de picaduras. Refiere caída desde su plano de sustentación y posterior cervicalgia.

En la exploración física presenta frecuencia cardiaca de 71 latidos por minuto, presión arterial de 130/71 mmHg, frecuencia respiratoria de 18 respiraciones por minuto y oximetría de pulso del $94 \%$. Muestra

\section{Correspondencia:}

*León F. Mendoza-Vega

E-mail: dr.mendozaleon@gmail.com
Disponible en internet: 07-03-2022

Fecha de recepción: 14-11-2021

Fecha de aceptación: 10-12-2021 DOI: 10.24875/REIE.2100015
Rev Educ Investig Emer. 2022;4(Supl 1):63-66 www.medicinadeemergencias.com bajo la licencia CC BY-NC-ND (http://creativecommons.org/licenses/by-nc-nd/4.0/). 


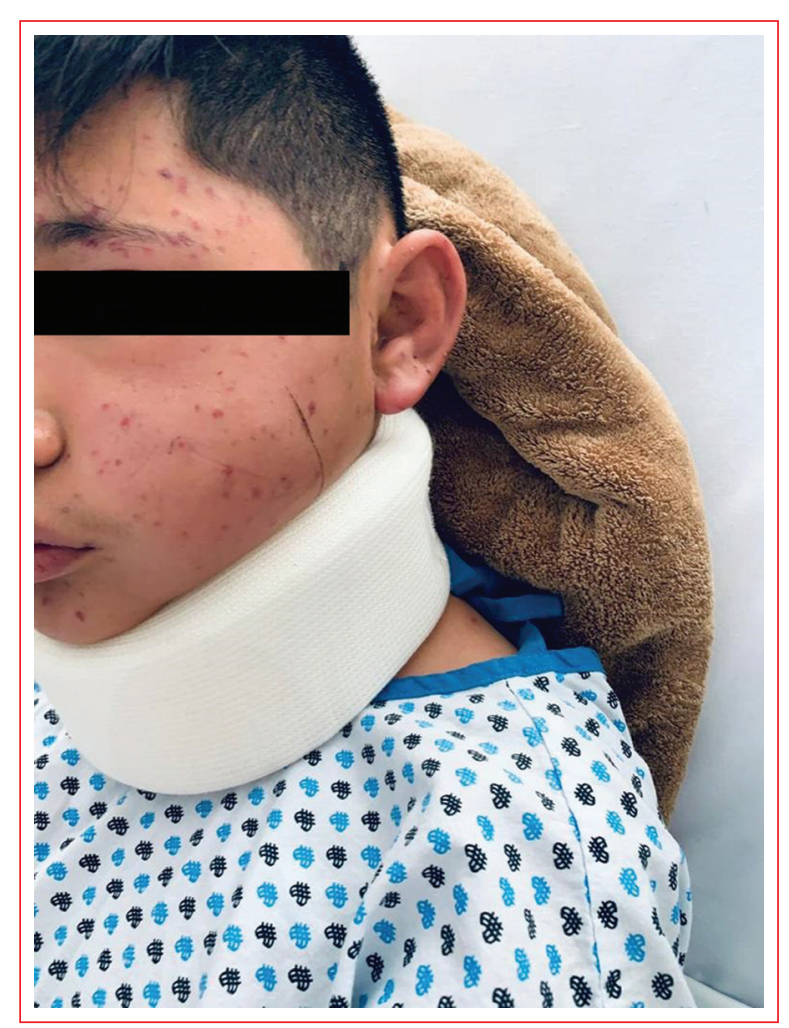

Figura 1. Edema facial y zona de eritema en la región malar izquierda.

edema facial y una zona de eritema en la región malar izquierda, dolor a la movilización de cuello y sin dolor a nivel cervical posterior (Fig. 1). En el tórax hay múltiples lesiones maculares sin aguijón, campos pulmonares bien ventilados, ruidos cardiacos rítmicos de buena intensidad y frecuencia, y abdomen sin compromiso (Figs. 2 y 3), sin más alteraciones.

Los paraclínicos a su ingreso revelan: sodio sérico 142 $\mathrm{mEq} / \mathrm{l}$, potasio sérico $4.5 \mathrm{mEq} / \mathrm{l}$, cloro $108 \mathrm{mEq} / \mathrm{l}$, calcio $8.6 \mathrm{mg} / \mathrm{dl}$, fosforo $5.5 \mathrm{mg} / \mathrm{dl}$, magnesio $1.67 \mathrm{mg} / \mathrm{dl}$, creatina cinasa MB $290.7 \mathrm{ng} / \mathrm{ml}$, creatina cinasa 15,381 UI/l, deshidrogenasa láctica (DHL) $1258 \mathrm{UI} / \mathrm{l}$, creatinina $1.31 \mathrm{mg} / \mathrm{dl}$, nitrógeno ureico en sangre (BUN) $27 \mathrm{mg} / \mathrm{dl}$, urea $57.78 \mathrm{mg} / \mathrm{dl}$ y glucosa $149 \mathrm{mg} / \mathrm{dl}$. El examen general de orina reporta: $\mathrm{pH} 5$, densidad 1.01, proteínas negativo, glucosa negativo, cuerpos cetónicos negativo, sangre $0.2 \mathrm{mg} / \mathrm{dl}$, leucocitos $4-6$ por campo y eritrocitos normomórficos 0-1 por campo.

Se integra falla renal aguda secundaria a rabdomiólisis y se inicia soporte hídrico con soluciones de base por Holliday-Segar con aumento de 1.5 de su requerimiento, sin potasio, más administración de antihistamínicos $\mathrm{AH} 1$ y esteroide, así como alcalinización de la orina.

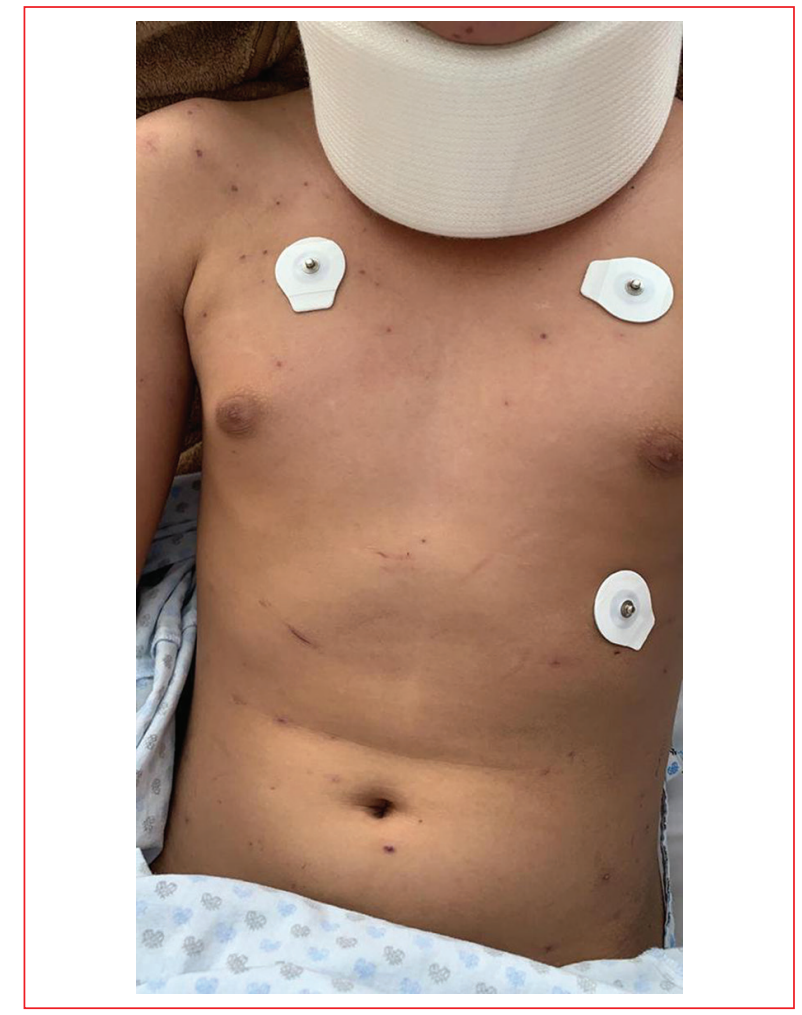

Figura 2. Tórax con múltiples lesiones maculares sin aguijón.

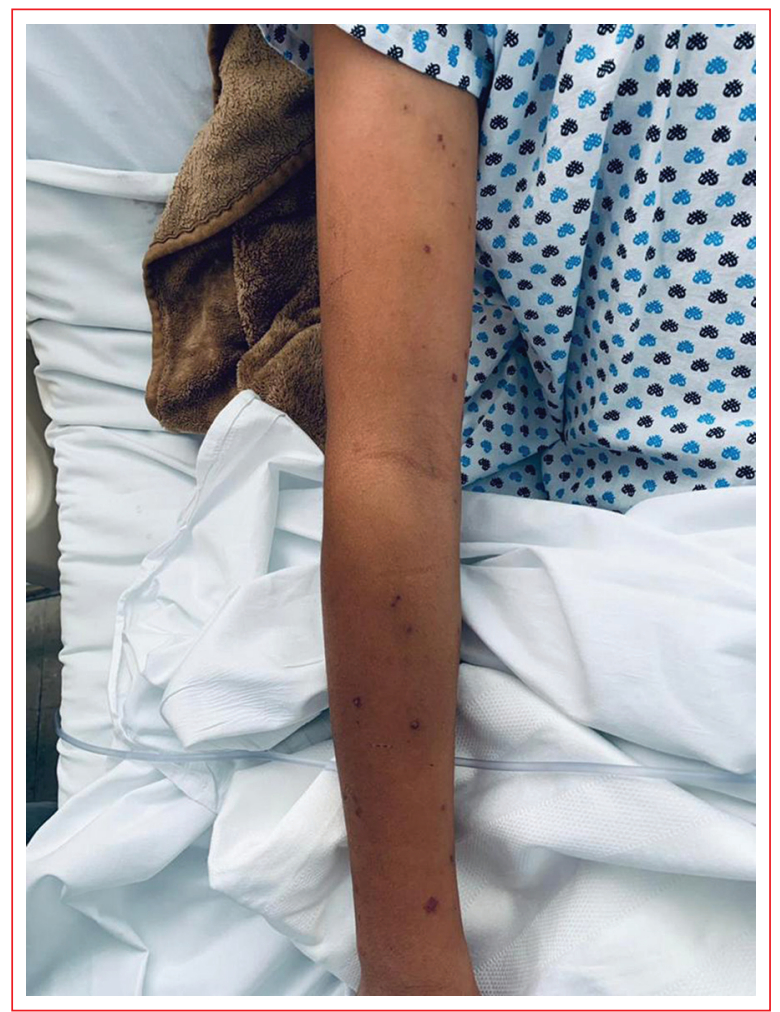

Figura 3. Máculas en las extremidades superiores. 
Los controles bioquímicos a las 12 horas reportan: sodio $138 \mathrm{mEq} / \mathrm{l}$, potasio $3.9 \mathrm{mEq} / \mathrm{l}$, cloro $109 \mathrm{mEq} / \mathrm{l}$, calcio $7.6 \mathrm{mg} / \mathrm{dl}$, fosforo $4.3 \mathrm{mg} / \mathrm{dl}$, magnesio $1.46 \mathrm{mg} / \mathrm{dl}$, creatina cinasa MB $396.9 \mathrm{ng} / \mathrm{ml}$, creatina cinasa 22,850 Ul/, DHL 1390 Ul/l, creatinina 0.98 mg/dl, BUN 26 mg/dl, urea $55.64 \mathrm{mg} / \mathrm{dl}$ y glucosa $117 \mathrm{mg} / \mathrm{dl}$.

Los controles a las 24 horas muestran: sodio sérico $145 \mathrm{mEq} / \mathrm{l}$, potasio sérico $3.4 \mathrm{mEq} / \mathrm{l}$, cloro $113 \mathrm{mEq} / \mathrm{l}$, calcio $7 \mathrm{mg} / \mathrm{dl}$, fosforo $3.8 \mathrm{mg} / \mathrm{dl}$, magnesio $1.32 \mathrm{mg} / \mathrm{dl}$, creatina cinasa MB $346.4 \mathrm{ng} / \mathrm{ml}$, creatina cinasa 24,272 UI//, DHL $1502 \mathrm{UI} / \mathrm{l}$, creatinina $0.82 \mathrm{mg} / \mathrm{dl}$, BUN $17 \mathrm{mg} / \mathrm{dl}$, urea $36.38 \mathrm{mg} / \mathrm{dl}$ y glucosa $116 \mathrm{mg} / \mathrm{dl}$.

Los controles a las 36 horas reportan: creatina cinasa MB $257.9 \mathrm{ng} / \mathrm{ml}$, creatina cinasa 19,256 UI/l y DHL $1147 \mathrm{UI} / \mathrm{l}$.

Se vigiló la función renal manteniendo el gasto urinario hasta alcanzar $154 \mathrm{ml} / \mathrm{m}^{2} \mathrm{schr}$. Se reportó elevación de las enzimas hepáticas (alanino aminotransferasa y aspartato aminotransferasa), por lo que se inició vitamina $\mathrm{E}$ como medida hepatoprotectora. Al tercer día de estancia hospitalaria se mantiene con evolución clínica favorable, se decide el cambio de aporte hídrico por vía oral y se le egresa a su domicilio.

Los controles al egreso reportan: sodio $142 \mathrm{mEq} / \mathrm{l}$, potasio $3.6 \mathrm{mEq} / \mathrm{l}$, cloro $110 \mathrm{mEq} / \mathrm{l}$, calcio $7.5 \mathrm{mg} / \mathrm{dl}$, creatina cinasa MB $105.6 \mathrm{ng} / \mathrm{ml}$, creatina cinasa $6180 \mathrm{UI} / \mathrm{l}, \mathrm{DHL} 629 \mathrm{UI} / \mathrm{l}$, aspartato aminotransferasa 226, creatinina $0.65 \mathrm{mg} / \mathrm{dl}$, BUN $14 \mathrm{mg} / \mathrm{dl}$, urea $29.96 \mathrm{mg} / \mathrm{dl}$ y glucosa $140 \mathrm{mg} / \mathrm{dl}$. El examen general de orina informa: $\mathrm{pH} 7.5$, densidad 1.01, proteínas negativo, cuerpos cetónicos negativo, sangre negativo y leucocitos 8-12 por campo.

\section{Discusión}

México es un productor importante de miel en América Latina, lo que predispone a un mayor riesgo de ataques en comunidades apícolas. El promedio anual de muertes por picadura de abeja fue de 21 en el periodo de 1988 a 2009, con más mortalidad en los mayores de $50 a^{n}{ }^{8} s^{8}$. La intoxicación masiva se presenta con una cantidad de picaduras superior a 500-1000 en adultos. Dada la menor superficie corporal con respecto a la distribución del veneno y la incapacidad para escapar de los ataques, se considera a los pacientes pediátricos con mayor riesgo de presentar una respuesta multisistémica con menor exposición al ataque.

Los casos reportados por Cahyani et al. ${ }^{9}$ incluyen un paciente de 15 años con lesión renal posterior a 30 picaduras de avispas y un paciente de 12 años que presentó falla multiorgánica secundaria a un ataque de 80 picaduras de avispa. En nuestro paciente no se sabe la cantidad específica de picaduras, debido al manejo antihistamínico y antiinflamatorio administrado previamente en el hospital de referencia, sin permitir valorar la totalidad de las lesiones individualmente.

Al ingreso presentaba edema facial y lesiones inflamatorias, con predominio en el tórax. A las 22 horas del ataque se reportan laboratorios con elevación de la creatina cinasa MB $(290.7 \mathrm{ng} / \mathrm{ml})$, creatina cinasa (15,381 UI/I), DHL (1258 UI/I), creatinina $(1.31 \mathrm{mg} / \mathrm{dl})$, BUN $(27 \mathrm{mg} / \mathrm{dl})$ y urea $(57.78 \mathrm{mg} / \mathrm{dl})$, con un pico máximo de creatina cinasa $(22,850 \mathrm{UI})$ a las 48 horas. Concordamos con lo reportado por Mejía-Vélez en $2010^{10}$, con instauración de la lesión renal aguda en un promedio de 2 días en los 48 casos descritos en su estudio (seis pacientes menores de 15 años). Así mismo, la DHL se elevó en 48 horas, alcanzando 1502 UI/I. Dados los efectos proinflamatorios y las lesiones hepatocelulares descritos con el veneno de abejas africanas, se administró vitamina $E$ por su efecto hepatoprotector ${ }^{11,12}$. A los péptidos del veneno se les atribuye mionecrosis e inducción de apoptosis secundarias a la señalización de caspasas. La hepatomegalia o la sensibilidad en el cuadrante superior derecho se han descrito, pero nuestro paciente no las presentó ${ }^{13}$.

La principal causa desencadenante de rabdomiólisis en menores de 10 años son las infecciones virales, como influenza, Coxsackie y virus de la inmunodeficiencia humana. En los adolescentes predominan las causas traumáticas. La picadura de abejas es una causa excepcional y poco reportada en pacientes pediátricos ${ }^{14}$.

La lesión renal aguda se atribuye a la rabdomiólisis y la hemólisis. Se ha comprobado el efecto de las fosfolipasas A1 y A2 como causante de la rabdomiólisis. En los adultos incluso amerita terapia de sustitución renal ${ }^{15}$.

El tratamiento se basa en corregir la hipovolemia, mejorar la isquemia renal, favorecer el aclaramiento de tóxicos, y prevenir y tratar los efectos adversos causados por las toxinas del veneno ${ }^{15}$.

Esas intervenciones se realizaron en nuestro paciente, quien al tercer día de tratamiento hídrico intensivo logró disminuir las enzimas musculares y las pruebas de función hepática, con mejoría en la diuresis diaria, y fue egresado sin secuelas.

\section{Conclusiones}

Las picaduras de abejas rara vez causan problemas de salud graves; cuando estos se producen, están 
relacionados con la anafilaxia. Las picaduras múltiples representan un riesgo de anafilaxia, rabdomiólisis y falla renal aguda secundaria; condiciones que el médico debe tener en mente para realizar un abordaje intencionado, detectar el problema e iniciar el manejo adecuado para evitar la lesión renal.

\section{Agradecimientos}

Al empuje y la motivación que nos da la presencia de profesionales jóvenes y con ganas de mejorar la medicina en México. A los/las residentes del Hospital Infantil de Tlaxcala.

\section{Financiamiento}

No se recibió ningún financiamiento para este trabajo.

\section{Conflicto de intereses}

Los autores declaran no tener ningún conflicto de intereses.

\section{Responsabilidades éticas}

Protección de personas y animales. Los autores declaran que para esta investigación no se han realizado experimentos en seres humanos ni en animales.

Confidencialidad de los datos. Los autores declaran que han seguido los protocolos de su centro de trabajo sobre la publicación de datos de pacientes.
Derecho a la privacidad y consentimiento informado. Los autores han obtenido el consentimiento informado de los pacientes y/o sujetos referidos en el artículo. Este documento obra en poder del autor de correspondencia.

\section{Bibliografía}

1. Bezerra DS, Gomes VA, Timbó RM, Ribeiro VV, Barros NJ, Sampaio FJ, et al. Acute injury complicating bee stings - a review. Rev Inst Med Trop Sao Paulo. 2017;59:e25.

2. Fitzgerald KT, Flood AA. Hymenoptera stings. Clin Tech Small Anim Pract. 2006;21:194-204.

3. Vikrant S, Jaryal A, Gupta D., Parashar A. Epidemiology and outcome of acute kidney injury due to venomous animals from a subtropical region of India. Clin Toxicol. 2019;57:240-5.

4. Vikrant S, Parashar A. Acute kidney injury due to multiple Hymenoptera stings - a clinicopathological study. Clin Kidney J. 2017;10:532-8.

5. Hughes RL. A fatal case of acute renal failure from envenoming syndrome after massive bee attack. A case report and literature review. Am J Forensic Med Pathol. 2019;40:52-7.

6. Chen TH, Liao WT, Chen CS, Lin PC, Wu MY. An envenoming syndrome from massive vespa stings induces multiple organ failure, case report. Insects. 2020;11:219.

7. Bridi RA, Blabi AL, Neves PM, Ponce D. Acute kidney injury after massive attack of Africanised bees. BMJ Case Rep. 2014;2014:bcr2013201381.

8. Becerril-Ángeles M, Núñez-Velázquez M, Arias-Martínez MI. Mortality related to honey-bee stings in Mexico from 1988 to 2009. Revista Alergia Mexico. 2013;60:58-62.

9. Cahyani GA, Risti MS, Meilania S, Partini PT. Delayed admission and management of pediatric acute kidney injury and multiple organ dysfunction syndrome in children with multiple wasp stings: a case series. Case Rep Nephrol Dial. 2019;9:137-48.

10. Mejía-Vélez G. Acute renal failure due to multiple stings by Africanized bees. Report on 43 cases. Nefrologia. 2010;30:531-8.

11. Betten DP, Richardson MD, Tong TC, Clark RF. Massive honeybee envenomation-induced rhabdomyolysis in an adolescent. Pediatrics. 2006; $117: 231-5$

12. Adikwu E, Nelson B. Hepatoprotective effect of vitamin E. Am J Pharmacol Toxicol. 2012;7:154-63.

13. Thiru T, Bak LG, Chee LL, Lai ML, Si YT. Acute renal failure following multiple wasp stings. Nephrol Dial Transplant. 1999;14:214-7.

14. Gong J, Yuan H, Gao Z, Hu F. Wasp venom and acute kidney injury: The mechanisms and therapeutic role of renal replacement therapy. Toxicon. 2019;163:1-7.

15. Chun-Yu C, Yan-Ren L, Lu-Lu Z, Wen-Chieh Y, Yu-Jun C, Kang-Hsi W et al. Clinical spectrum of rhabdomyolysis presented to pediatric emergency department. BMC Pediatr. 2013;13:134 